\title{
Complex Carbohydrate-Lectin Interaction at the Interface: a Model for Cellular Adhesion. II. Beactivity of both the Oligosaccharide Chain and Sugar-binding Domain of a Glycoprotein Lectin
}

\author{
Abhijit Chakrabarti† and Sunil K. Podder ${ }^{\star}$ \\ Department of Biochemistry, Indian Institute of Science, Bangalore. India
}

\begin{abstract}
We describe studies of a new model cell adhesion system involving liposomes bearing lectins and the glycosphingolipid, asialomonosialoganglioside (asialoGM ${ }_{1}$ ). The model provides a simple analysis of experimental data to elucidate the mechanism of heterophilic cell-cell adhesion mediated by multiple protein-carbohydrate interactions. Phospholipid vesicles bearing the fatty acid conjugate of a glycoprotein lectin from Ricinus communis (RCAI vesicle) are shown to react with vesicles bearing the fatty acid conjugate of Concanavalin $A$ (Con $A$ ) and asialoGM ${ }_{1}$ (Con $A$ vesicle). The kinetics of aggregation and monosaccharide-induced disaggregation of the two types of vesicles were followed by monitoring the time-dependent change in turbidity. Depending on the surface density of the asialoGM ${ }_{1}, 40-60 \%$ of the resulting precipitin complex was dissociable only in the presence of both RCAI-specific galactose and Con A-specific $\alpha$-methyl-D-mannoside. Results indicate simultaneous participation of both the saccharide-binding domain and carbohydrage sequence of RCAI, a model cell adhesion molecule, to stabilize the encounter complex by two types of interactions. These findings support the possibility of stable cell-cell adhesion in vivo occurring via interactions between cell adhesion molecules on apposing cell-surface membranes.
\end{abstract}

\section{INTRODUCTION}

Cell adhesion molecules (CAM) are cell-surface glycoproteins. Carbohydrate chains modulate adhesion as found in the case of the neural cell adhesion molecule (NCAM) where the extent of sialylation affected the rate of homophilic NCAM-NCAM interaction when isolated at two different developmental stages (Hoffman and Edelman, 1983). The cDNA-derived amino acid sequences show multiple structural domains in CAM (Edelman, 1988; Stoolman, 1989), one of which is lectin-like. Amino acid sequences of adhesion molecules controlling lymphocyte migration e.g., MEL-14 antigen, GMP-140 and ELAM-1 (Bowen et al., 1990; Johnston et al., 1989; Hession et al., 1990) reveal a lectin-like domain. A large body of data implicates a family of receptors, the selectins (lectin-complement binding (AM), in the initial interactions between leukocytes and vascular endothelia (Lawrence and Springer, 1991; Watson et al., 1991). Direct functioning of the lectin-like domain is first evidenced in the lymphocyte homing receptor, where an anionic polysaccharide is shown to play a role in lymphocyte adhesion (Bowen et al., 1990; Coombe and Rider, 1989). Recently, the three members of the selectin receptor family have been shown to recognize a common carbo-

\footnotetext{
- Author to whom correspondence should be addressed. $\dagger$ Present address: Department of Biology, The Johns Hopkins University, 3400 N. Charles Street, Baltimore. MD 21218, USA.

Abbreviations used: CAM, cell adhesion molecules: NCAM, neural cell adhesion molecule; DMPC, dimyristoylphosphatidylcholine; Gal, galactose; Me-Mann, $\alpha$-methyl-D-mannopyranoside; asialoGM . asialomonosialoganglioside; RCAI, Ricinus communis agglutinin: Con A. Concanavalin A; PNA, peanut agglutinin.
}

hydrate epitope, the sialylated Lewis ${ }^{x}$ oligosaccharide (Foxall et al., 1992).

We have designed a liposomal system bearing complementary lectin and carbohydrate molecules to study the molecular mechanism of heterophilic cell-cell adhesion. The kinetics of the aggregation of these two types of vesicles were interpreted in the light of cellular adhesion mediated by a single specific proteincarbohydrate interaction (Chakrabarti and Podder, 1990). The present study shows the reactivity of both the carbohydrate-reactive domain and the oligosaccharide chain of the glycoprotein lectin, a model CAM, involved in multiple interactions in liposomes.

\section{EXPERIMENTAL}

Dimyristoylphosphatidylcholine (DMPC), sodium cholate, octyl- $\beta$-D-glucopyranoside, galactose $(\mathrm{Gal}), \alpha$ methyl-D-mannopyranoside (Me-Mann), cholesterol and latex particles $(305 \mathrm{~nm}$ diameter) were obtained from Sigma (St Louis, MO, USA). DEAE-Sephadex, Sephadex G-100, Sepharose 4B and Sephacryl S-1000 were obtained from Pharmacia Co. (Uppsala, Sweden). The other chemicals used were all of analytical grade and locally available.

Asialomonosialoganglioside (asialoGM ${ }_{1}$ ) was prepared by hydrolysing a mixture of di- and trisialogangliosides with $1.0 \mathrm{~m}$ formic acid and collected after passing through the same DEAE-Sephadex column with methanol as discussed earlier (Chakrabarti and Podder, 1990).

Ricinus communis agglutinin (RCAI) was isolated from castor seeds using DEAE-Sephadex followed by 


\begin{tabular}{|c|c|c|c|}
\hline $\begin{array}{l}\text { Vesicles } \\
\text { Latex particle }\end{array}$ & $\begin{array}{l}\text { Diameter }(n \mathrm{~nm}) \\
\quad 305\end{array}$ & $\begin{array}{c}\text { Molecular weights }(\mathrm{Da}) \\
-\end{array}$ & $\begin{array}{c}K_{D}^{a} \\
0.063\end{array}$ \\
\hline $\begin{array}{l}\text { Sonicated vesicles } \\
\text { of egg yolk lecithin }\end{array}$ & 25 & $2.06 \times 10^{6}$ & 0.704 \\
\hline \multicolumn{3}{|l|}{$\begin{array}{l}\text { DMPC with } 33 \% \text { cholesterol } \\
\text { prepared by the dialvsis }\end{array}$} & 0.327 \\
\hline $\begin{array}{l}\text { DMPC with fatty acid } \\
\text { conjugate of RCAI }\end{array}$ & 300 & $2.21 \times 10^{8}$ & 0.068 \\
\hline \multicolumn{3}{|l|}{ DMPC with fatty acid } & 0.423 \\
\hline \multicolumn{4}{|c|}{$\begin{array}{l}\text { a } K_{\mathrm{D}} \text { is the partition coefficient given by }\left(V_{\mathrm{e}}-V_{0}\right) /\left(V_{\mathrm{t}}-V_{0}\right) \text {, where } V_{0} \text { is the } \\
\text { void volume }(16 \mathrm{~mL}) \text { and } V_{\mathrm{t}} \text { is the total volume }(38 \mathrm{~mL}) \text { of the Sephacryl } \\
\mathrm{S}-1000 \text { column and } V_{\mathrm{e}} \text { is the elution position of the sample. } \\
\text { "From Huang }(1969) \\
\text { c Determined from the plot of diameter and molecular weight data } \\
\text { published by Zumbuehl and Weder }(1981)\end{array}$} \\
\hline
\end{tabular}

Sephadex G-100 column chromatography (Lappi et al., 1978). Concanavalin A (Con A) was isolated from Jack bean meal by affinity chromatography (Agrawal and Goldstein, 1967). Peanut agglutinin (PNA) was isolated from locally available seeds (Arachis hypogaea) using lactamylsepharose $4 \mathrm{~B}$ affinity column chromatography (Maiti and Podder, 1989). Protein concentrations were determined spectrophotometrically at $\mathrm{pH} 7.4$ in a buffer containing $10 \mathrm{~mm}$ Tris- $\mathrm{HCl}, 0.1 \mathrm{M}$ $\mathrm{NaCl}$ using an absorbance $\left(A_{280 \mathrm{~nm}}^{1 \%}\right)$ of 11.8 for RCAI, 11.4 for Con $\mathrm{A}$ and 7.7 for PNA.
Preparation of the conjugated lectins and the two types of vesicles have been described previously (Chakrabarti and Podder, 1990). Briefly, the lectins were conjugated with stearoyl chloride in a buffer containing sodium cholate $(2 \%)$. Phospholipid vesicles were made by the dialysis of cholate from a mixture of the conjugated proteins and lipids. AsialoGM $\mathrm{M}_{1}$ was cosolubilized with DMPC in chloroform and dried under a stream of nitrogen.

The rate constants were determined from the slope of the linear semilogarithmic plots of the change in

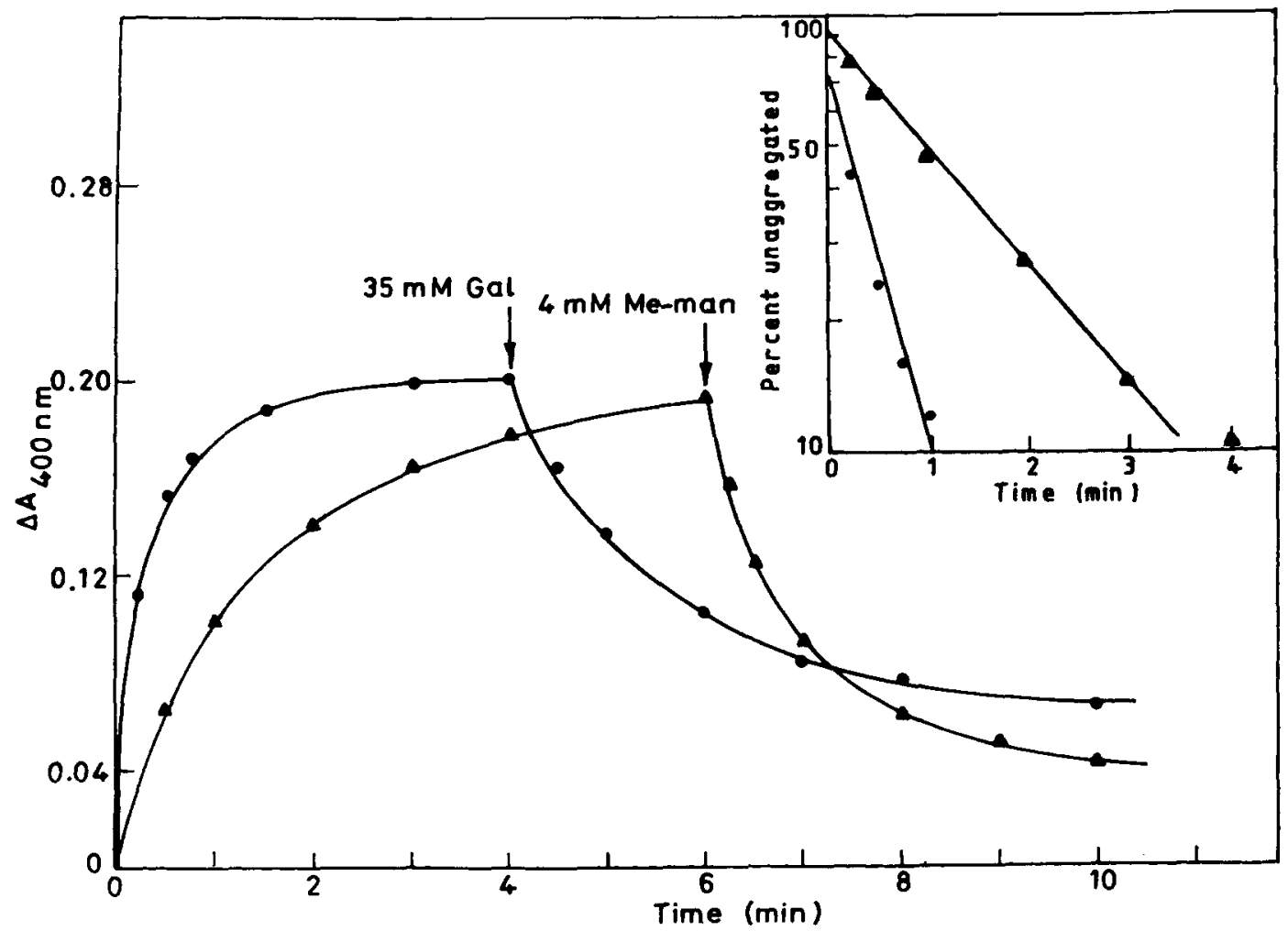

Figure 1. Kinetics of aggregation of two types of vesicles. Con A vesicles bearing $0.1 \%$ fatty acid conjugate of Con $A$ and $25 \%$ asialoGM, with the lectin vesicle bearing fatty acid conjugate of PNA (O) and aggregation between vesicle-bound Con A and RCAI $(\triangle)$. Arrow indicates addition of dissociating monosaccharidess. The inset shows the semilogarithmic plot of the aggregation profile. Concentrations of the reacting components are given in Table 2. 


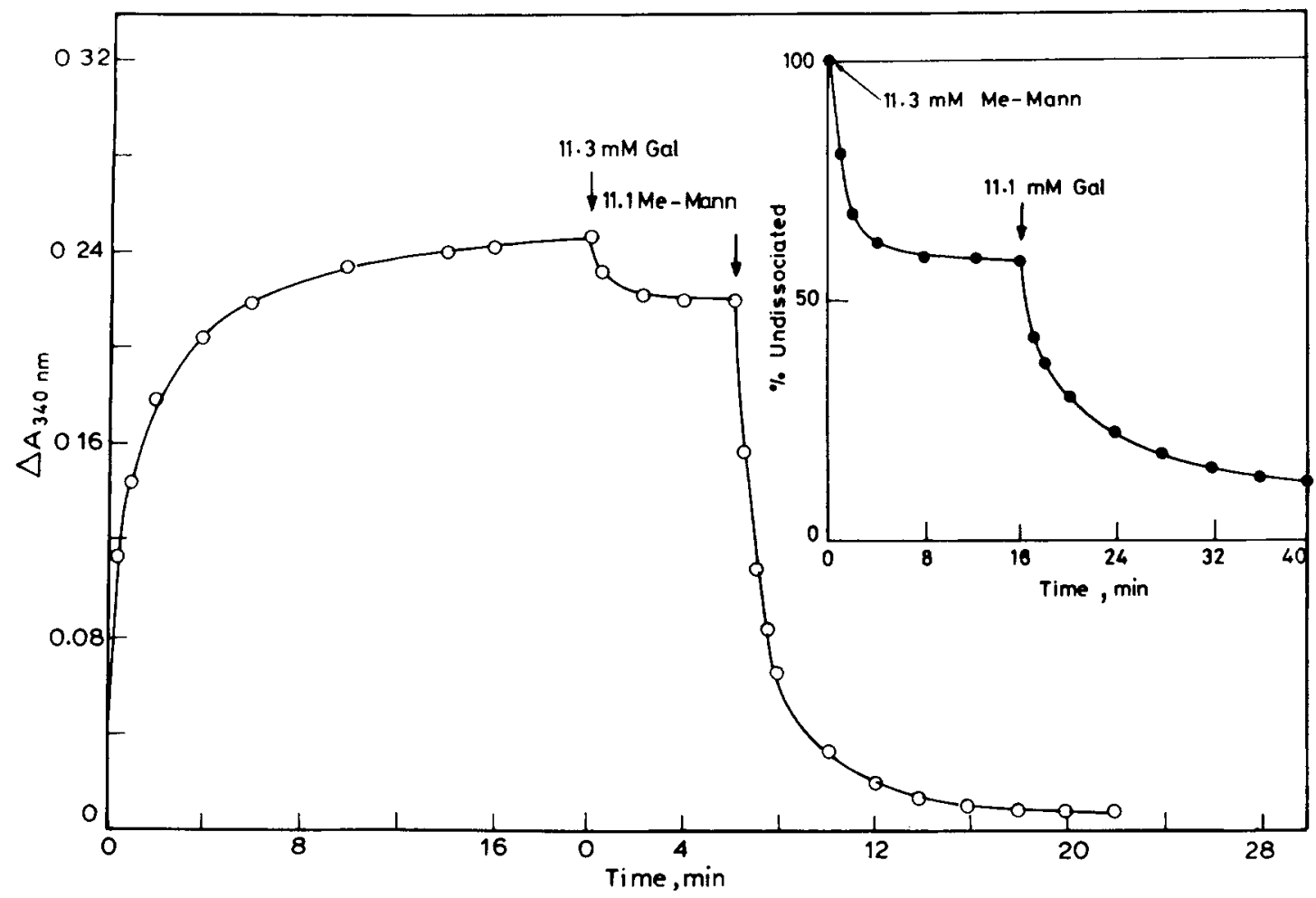

Figure 2. Kinetics of aggregation between RCAl vesicles and Con $A$ vesicles bearing $5 \%$ asialoGM in addition to $0.1 \%$ Con A. Arrow indicates addition of dissociating monosaccharides. Inset shows the dissociation profile when the order of addition of the two monosaccharides was reversed. In both cases the asialoGM $\mathrm{M}_{1}$ content of the vesicles is the same.

absorbance with time both for the association and sugar-induced dissociation processes under pseudo-first order reaction conditions (Chakrabarti and Podder, 1990).

The change in turbidity was measured in a Shimadzu UV-190 double beam spectrophotometer equipped with a U-135 recorder at a wavelength of $340 \mathrm{~m}$ or $400 \mathrm{~nm}$. The $90^{\circ}$ light scattering was measured in a Hitachi-650-60 spectrofluorimeter at $400 \mathrm{~nm}$.

\section{Characterization of vesicles}

Average diameters and molecular weights of RCAI and Con $\mathrm{A}$ vesicles (with and without asialoGM $\mathrm{G}_{1}$ ) were determined by gel filtration on a Sephacryl S-1000 column $(48 \times 1 \mathrm{~cm})$ according to the method described by Reynolds et al. (1983). The liposome-containing fractions were detected by measuring the $90^{\circ}$ light scattering in a spectrofluorimeter. The column was calibrated with (i) latex particles ( $305 \mathrm{~nm}$ diameter) and (ii) sonicated vesicles of egg yolk lecithin $(25 \mathrm{~nm}$ diameter). The sonicated egg phosphatidylcoline vesciles $\left(2.06 \times 10^{6} \mathrm{Da}\right)$, as described by Huang (1969), and DMPC vesicles containing $33 \%$ cholesterol $\left(1.2 \times 10^{8} \mathrm{Da}\right)$ were used as the molecular weight markers.

Table 1 lists the molecular weights and diameters of the different vesicles determined from the elution profile on the Sephacryl S-1000 column. Liposomes with asialoGM $M_{1}$ alone were smaller ( $30 \mathrm{~nm}$ diameter), and were prepared by bath sonication.

For vesicles containnig RCAI and Con A and/or asialoGM $M_{1}$, average diameters were found to be 300 and $150 \mathrm{~nm}$ with molecular weights of $2.21 \times 10^{8}$ and $9.8 \times 10^{7} \mathrm{Da}$, respectively.

Table 2. Rate constants of formation $\left(k_{f}\right)$ and dissociation $\left(k_{d}\right)$ of the aggregation reaction between two types of DMPC vesicles containing complementary protein and carbohydrate molecules

\begin{tabular}{|c|c|c|c|c|}
\hline Vesicle 1 & Vesicle 2 & $\begin{array}{l}k_{f}\left(\times 10^{-7}\right) \\
{[/ M(\text { ves. }) s]}\end{array}$ & $\begin{array}{c}k_{d}\left(\times 10^{3}\right)^{a} \\
(/ / s)\end{array}$ & $\begin{array}{c}K_{\text {eq }}\left(\times 10^{-10}\right)^{\mathrm{b}} \\
(/ \mathrm{M})\end{array}$ \\
\hline CAl $(2.1 \mu \mathrm{M})$ & AsialoGM $1(12.4 \mu \mathrm{M})$ & 1.71 & 2.090 & 0.82 \\
\hline CAI & ) & 6 & & 4.44 \\
\hline NA $(0.29 \mu \mathrm{M})$ & $\begin{array}{l}\text { AsialoGM }(185 \mu \mathrm{M}) \\
\text { Con } \mathrm{A}(1.3 \mu \mathrm{M})\end{array}$ & 2.62 & 0.245 & 10.69 \\
\hline \multicolumn{5}{|c|}{$\begin{array}{l}\text { The } k_{\mathrm{d}} \text { values were calculated using values for the equilibrium constant of } \\
1.4 \times 10^{4} / \mathrm{M} \text { for the Con A-mannose system (Podder et al., 1974) and } \\
0.94 \times 10^{3} / \mathrm{M} \text { (Neurohr et al., 1981) for the PNA-Gal system. In all cases, the } \\
\text { densities of the conjugated proteins were kept constant at } 0.1 \% \text { with respect } \\
\text { to DMPC. } \\
{ }^{b} \mathrm{~K}_{\mathrm{eq}}=\mathrm{k}_{\mathrm{f}} / \mathrm{k}_{\mathrm{d}} \text {. }\end{array}$} \\
\hline
\end{tabular}


Table 3. Extent of sugar-induced dissociation of aggregates formed by the RCAI vesicle and Con $A\left(\right.$ asialoGM $M_{1}$ ) vesicle

\begin{tabular}{llccc} 
RCAl vesicle & Con A vesicle & \multicolumn{2}{c}{ Concentration of sugars } & Dissociation \\
RCAl $(0.25 \mu \mathrm{M})$ & Me-Man $(\mathrm{mM})$ & Gal (mM) & $(\%)$ \\
& Con A $(1.5 \mu \mathrm{M})$ & 11.3 & - & $44.0(34)$ \\
& AsialoGM & 11.1 & 11.1 & $48.0(140)$ \\
& $(105 \mu \mathrm{M})$ & - & 11.3 & $10.0(<10)$ \\
& & 11.1 & 11.1 & $88.0(58)$ \\
a Values in parentheses are $t_{0.5}$ values & &
\end{tabular}

\section{RESULTS}

Con A did not hinder accessibility of asialoGM $M_{1}$ in phospholipid vesicles (Con A vesicle). They reacted with vesicles bearing the fatty acid conjugate of PNA, a Gal-binding protein, but not a glycoprotein, instead of RCAI. Figure 1 shows the time-dependent change in absorbance due to the aggregation and monosaccharide-induced disaggregation of (i) PNA vesciles and Con $A$ vesicles bearing asialoGM $M_{1}$ and (ii) RCAI vesicles with the Con A vesicle in the absence of asialoGM $M_{1}$. The presence of $0.1 \mathrm{~mol} \%$ Con $A$ in the Con $A$ vesicle did not mask asialoGM $\mathrm{G}_{1} \mathrm{~s}$, and PNA vesicles reacted with them giving rise to a precipitin reaction. The addition of Gal decreased absorbance dissociating $>80 \%$ of the total complex. Similarly vesicle-bound RCAI and Con A also caused precipitation, dissociable by Me-Mann showing the specificity of interaction (Fig. 1). The semilogarithmic plot of these associations was characterized by a single time constant as shown in the inset in Fig. 1.

Figure 2 shows the change in absorbance with time upon aggregation of the RCAI vesicle and the Con A vesicle containing $5 \mathrm{~mol} \%$ asialo $\mathrm{GM}_{1}$ with respect to DMPC and the monosaccharide-induced disaggregation. The time-dependent disaggregation profiles,

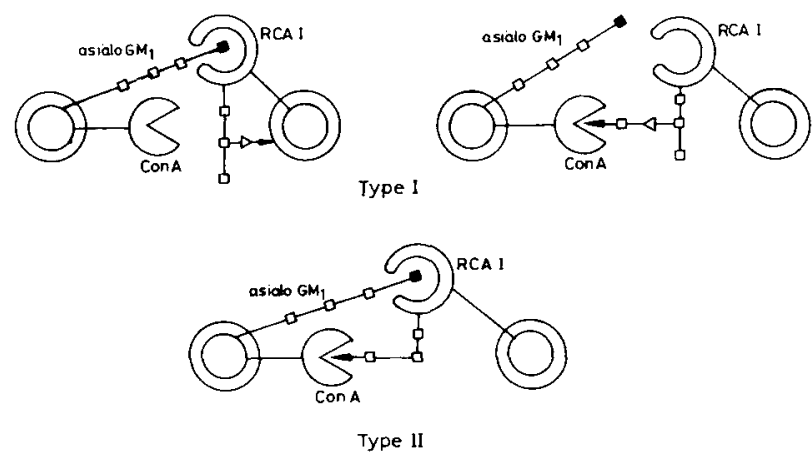

Figure 3. Schematic representation of the two types of complexes as detected by the distinct differences in the kinetics of monosaccharide-induced dissociation.

Table 4. Density-dependent formation of Type II complexes

\begin{tabular}{|c|c|c|c|}
\hline RCAI vesicle & Con A vesicle & $\begin{array}{l}\text { Density of } \\
\text { asialoGM } \\
(\%)\end{array}$ & $\begin{array}{c}\text { Type It complex } \\
(\%)\end{array}$ \\
\hline $\operatorname{RCAl}(0.25 \mu \mathrm{M})$ & $\begin{array}{l}\text { Con A }(1.5 \mu \mathrm{M}) \\
\text { AsialoGM } 1(105 \mu \mathrm{M})\end{array}$ & 5.0 & 40.0 \\
\hline $\operatorname{RCAl}(0.26 \mu \mathrm{M})$ & $\begin{array}{l}\text { Con A }(1.45 \mu \mathrm{m}) \\
\text { AsialoGM }(360 \mu \mathrm{M})\end{array}$ & 10.0 & 45.0 \\
\hline $\operatorname{RCAl}(0.30 \mu \mathrm{M})$ & $\begin{array}{l}\text { Con A }(1.1 \mu \mathrm{M}) \\
\text { AsialoGM }_{1}(540 \mu \mathrm{M})\end{array}$ & 16.0 & 60.0 \\
\hline
\end{tabular}

induced by the individual monosaccharides, Gal and Me-Mann, show remarkable differences in the kinetic properties. Even though asialoGM $M_{1}$ is present in a 50 -fold excess over Con $\mathrm{A}$, only $10-15 \%$ of the total complex is dissociable by Gal. The inset shows the differences both in the extent and time constants of the dissociation processes when the order of the addition of the respective monosaccharides was reversed. Moreover, the semilogarithmic plot of the association process is biphasic (data not shown) indicating the formation of two different types of complexes. Table 2 summarizes the rat constants and equilibrium constants of associations and monosaccharide-induced dissociations for the individual RCAI-Con A, PNA-asialoGM $M_{1}$ and RCAI-asialoGM $\mathrm{R}_{1}$ systems.

From the differences in the extent of dissociation by Gal and Me-Mann two types of complexes could be detected, as shown in Fig. 3 One type could be dissociated by either of the individual lectin-specific sugars, Me-Mann and Gal (Type I), and the other only in the presence of both (Type II). The exent of sugar-induced dissociation and the time (in seconds) corresponding to $50 \%$ total dissociation, $t_{05}$, brought about by the individual specific monosaccharide, is also remarkably different (Table 3).

The extent of formation of the Type II complex was determined from the differences of the extent of Gal-induced dissociation in the presence of methyl mannoside from the extent of formation in its absence. The extent of formation could also be determined from the difference of the extent of methyl mannosideinduced dissociations by reversing the order of additions of the respective monosaccharides (Table 3 ). The formation of Type II complexes, listed in Table 4, is the mean of two such determinations. Table 4 also shows the increase in Type II complex formation with increase in density of asialoGM $M_{1}$ in the Con $A$ vesicle.

\section{DISCUSSION}

The main objective in using liposomes carrying surfacebound complementary protein and carbohydrate molecules is to demonstrate the formation of multiple bonds leading to stable adhesion between two such surfaces. The formation of multiple bonds that strengthen adhesion between two apposing surfaces carrying complementary molecules could be achieved in two ways. More than one valency of the multifunctional ligand might be involved in binding as shown in the present study or a number of monovalent proteincarbohydrate bonds could form in order to stabilize the adduct (Chakrabarti and Podder, 1990). The model allows the simple estimation of contributions of indivi- 
dual carbohydrate-specific interactions, which is relevant for understanding the molecular mechanism of such processes.

Podder et al. (1974) showed that the mannose-rich oligosaccharide chains of RCAI were reactive to Con A giving rise to a precipitin reaction. We have studied the kinetics of the same reaction when RCAI and Con A were bound to DMPS liposomes. We added excess asialoGM $\mathrm{G}_{1}$ in Con $\mathrm{A}$ vesicles in order for the sugarbinding domain of RCAI to react. In the presence of 5$15 \mathrm{~mol}^{\%}$ asialoGM $\mathrm{G}_{1}$ in $\mathrm{Con} \mathrm{A}$ vesicles, an aggregate is formed where both the carbohyrate chains and the carbohydrate-reactive domain of the RCAI molecule are involved simultaneously in the formation of bonds. Figure 3 shows the possibility that RCAI can act as a model CAM in a reconstituted system by forming an adduct involving both the functional domains, the oligosaccharide chain and the carbohydrate-binding domain (Type II)

Assuming uniform distribution of Con $A$ and asialoGM $M_{1}$ on the spherical surface of the Con $A$ vesicle bearing asialoGM $\mathrm{M}_{1}$, we have calculated the surface density of asialoGM $\mathrm{G}_{1}$ to be $10^{5}$ per $\mu \mathrm{m}^{2}$ and $2 \times 10^{3}$ per $\mu \mathrm{m}^{2}$ for $\operatorname{Con} A$, and the average distance between Con $A$ and asialoGM $\mathrm{M}_{1}$ to be $14 \mathrm{~nm}$. This distance is too large for the formation of the Type II complex. This can only be achieved by the lateral diffusion and redistribution of components subsequent to the formation of the encounter complex. The formation of $35-40 \%$ Type II complex was favoured by the facile stereochemical orientation of Con $A$ anmd asialoGM $M_{1}$ on the surface of the vesicle.

An estimate of the relative stability of the bonds formed (Chakrabarti and Podder, 1990) is obtained from the ratio of $k_{f}$ and $k_{d}$ (see Table 2 ). The ratio $\left(K_{\mathrm{eq}}\right)$ is $\sim 10^{10}-10^{11} / \mathrm{M}$ in the formation of Type I complexes mediated by the individual complex carbohydratelectin interactions indicating the formation of multiple bonds.

\section{Acknowledgements}

Financial support from the Department of Atomic Energy (DAE) and Council of Scientific and Industrial Research (CSIR), India are gratefully acknowledged. One of us (AC) acknowledges a Senior Research Fellowship from CSIR, India.

\section{REFERENCES}

Agrawal, B. B. L. and Goldstein, I. J. (1967). Protein-carbohydrate interaction. VI. Isolation of Concanavalin A by specific adsorption on cross-linked dextran gels. Biochim. Biophys. Acta. 147, 262-271.

Bowen, B. R., Fennie, C. and Lasky, L. A. (1990). The MEL-14 antibody binds to the lectin domain of the murine peripheral lymh node homing receptor. J. Cell. Biol. 110, 147-153.

Chakrabarti, A. and Podder, S. K. (1990). Complex carbohydrate lectin interaction at the interface: a model for cellular adhesion. I. Effect of vesicle-size on the kinetics of aggregation between fatty acid conjugate of lectin and liposoma asialoganglioside. Biochim. Biophys. Acta. 1024, 103-110.

Coombe, D. R. and Rider, C. C. (1989). Lymphocyte homing receptors cloned-a role for anionic polysaccharides in lymphocyte adhesion. Immunol. Today 10, 289-291.

Edelman, G. M. (1988). Morphoregulatory molecules. Biochemistry 27, 3533-3543.

Foxall, C., Watson, S. R., Dowbenko, D., Fennie, C., Lasky, L. A. Kiso, M., Hasegawa, A., Asa, D. and Bradley, B. K. (1992) The three members of the selectin receptor family recognize a common carbohydrate epitope, the sialyl Lewis oligosaccharide. J. Cell. Biol. 117, 895-902.

Hession, C., Osborn, L., Goff, D., Chi-Rosso, G., Vassallo, C. Pasek, M., Pittack, C., Tizard, R., Goelz, S., McCarthy, K., Hopple, S. and Lobb, R. (1990). Endothelial leukocyte adhesion molecule. 1. Direct expression cloning and functional interactions. Proc. Natl Acad. Sci. USA 87, 1673-1677.

Hoffman, S. and Edelman, G. M. (1983). Kinetics of homophilic binding by embryonic and adult forms of the neural cel adhesion molecule. Proc. Natl Acad. Sci. USA 80, 57625766.

Huang, C. H. (1969). Studies on phosphatidylcholine vesciles Formation and physical characteristics. Biochemistry 8 , 344-351.

Johnston, G. I., Cook, R. G. and McEver, R. P. (1989). Cloning of GMP -140 , a granule membrane protein of platelets and endothelium: sequence similarly to proteins involved in cell adhesion and inflammation. Cell 56, 1033-1044.

Lappi, D. A., Kapmeyer, W., Beglau, J. M. and Kaplan, N. O. (1978). The disulphide bond connecting the chains of ricin. Proc. Natl Acad. Sci. USA 75, 1096-1100.

Lawrence, M. B. and Springer, T. A. (1991). Leukocytes roll on a selectin at a physiologic flow rates: distinction from and prerequisite for adhesion through integrins. Cell 65, 859873.

Maiti, T. K. and Podder, S. K. (1989). Differential binding of peanut agglutinin with lipopolysaccharides of homologous and heterologous Rizobium. FEMS Lett. 65, 279-284.

Neurohr, K. J., Young, N. M. Smith, I. C. P. and Mantsch, H. H. (1981). Kinetics of binding of methyl $\alpha-$ and $\beta-D-$ galactopyranoside to peanut agglutinin: a Carbon-13 nuclear magnetic resonance study. Biochemistry 20, 34993504.

Podder, S. K., Surolia, A. and Bachhawat, B. K. (1974). On the specificity of carbohydrate-lectin recognition: the interaction of a lectin from Ricinus communis beans with simple saccharides and Concanavalin A. Eur. J. Biochem. 44, 151160.

Reynolds, J. A., Nozaki, Y. and Tanford, C. (1983). Gel-exclusion chromatography on S-1000 Sephacryl: application to phospholipid vesicles. Anal. Biochem. 130, 471-474.

Stoolman, L. M. (1989). Adhesion molecules controlling lymphocyte migration. Cell 56, 907-910.

Watson, S. R., Imai, Y., Fennie, C., Geoffrey, J., Singer, M. Rosen, S. D. and Lasky, L. A. (1991). The complement binding-like domains of the murine homing receptor facilitate lectin activity. J. Cell. Biol. 115, 235-243.

Zumbuehl, O. and Weder, H. G. (1981). Liposomes of controllable size in the range of 40 to $180 \mathrm{~nm}$ by defined dialysis of lipid/detergent mixed micelles. Biochim. Biophys. Acta. 640. 252-262. 